\title{
Expression of CD40 is a positive prognostic factor of diffuse large B-cell lymphoma treated with R-CHOP (rituximab, cyclophosphamide, doxorubicin, vincristine, and prednisone)
}

This article was published in the following Dove Press journal:

OncoTargets and Therapy

23 June 2016

Number of times this article has been viewed

\section{Guoqi Song' \\ Huiyun $\mathrm{Ni}^{\prime}$ \\ Linqing Zou ${ }^{2}$ \\ Shukui Wang ${ }^{3}$ \\ Fuliang $\operatorname{Tian}^{4}$ \\ Hong Liu' \\ William C $\mathrm{Cho}^{5}$ \\ 'Department of Hematology, Affiliated Hospital of Nantong University, Nantong, ${ }^{2}$ Department of Human Anatomy, Nantong University, Nantong, ${ }^{3}$ Central Laboratory of Nanjing First Hospital, Nanjing Medical University, Nanjing, ${ }^{4}$ Maternal and Child Health Hospital of Lianyungang, Lianyungang, Jiangsu, People's Republic of China ${ }^{5}$ Department of Clinical Oncology, Queen Elizabeth Hospital, Kowloon, Hong Kong}

Correspondence: Hong Liu Department of Hematology, Affiliated Hospital of Nantong University, Room 6506, 5/F, 20 Xishi Road, Nantong, Jiangsu, People's Republic of China

Tel +86 I87 6I7| 3399

Fax +86 5I 281160506

Email hongliu1963@I63.com

William C Cho

Department of Clinical Oncology, Queen Elizabeth Hospital, Room I305, I3/F, Block R, 30 Gascoigne Road, Kowloon, Hong Kong

Tel +85235065441

Fax +8523506 5455

Email williamcscho@gmail.com
Objectives: The objective of this study was to investigate the expression level of CD40 and its role in the prognosis of patients with diffuse large B-cell lymphoma (DLBCL) who were treated with rituximab-CHOP (rituximab, cyclophosphamide, doxorubicin, vincristine, and prednisone).

Design and methods: The immunohistochemical expressions of CD40 in 186 well-characterized DLBCL patients were evaluated by tissue microarrays, thereby revealing the relationship of the molecule CD40 with known tumor, patient-related variables, and survival rates.

Results: The results showed that CD40 expressions were not statistically different between the germinal center B-cell-like (GCB) type and the non-GCB type. We also analyzed the relationships of CD40 expression with overall survival (OS) and progression-free survival (PFS) in DLBCL patients who were uniformly treated with R-CHOP. A low expression of CD40 compared to high expression is related to poor OS and PFS.

Conclusion: Our findings indicate that the CD40 level at onset acts as an independent prognostic predictor of DLBCL patients treated with R-CHOP.

Keywords: CD40, diffuse large B-cell lymphoma, R-CHOP, prognostic factor

\section{Introduction}

Diffuse large B-cell lymphoma (DLBCL) is one of the most common histological subtypes of non-Hodgkin's lymphoma, characterized by heterogeneity in its clinical, immunophenotypic, and genetic features. ${ }^{1}$ The discovery of rituximab and its correct application in combination with cyclophosphamide, doxorubicin, vincristine, and prednisone (CHOP) (standard first-line treatment) have significantly improved clinical outcome in DLBCL patients and have made the disease a curable malignant tumor. However, still a number of patients with DLBCL undergo relapse or show no response to the standard first-line therapy. ${ }^{2,3}$ Prediction of outcome is important to avoid overtreatment and also to identify others who might benefit from intensive therapy. As the most important prognostic factor for survival - International Prognostic Index (IPI) - has gained wide acceptance, it is considered to be the strongest indicator to identify high-risk patients and also as a guidance for individual treatment. ${ }^{4}$

The IPI, based on clinical features (eg, age, stage, and level of lactate dehydrogenase), neither reflects the molecular heterogeneity of DLBCL nor predicts responsiveness to therapy. The IPI score is no longer considered to be the strongest prognostic indicator in rituximab (R)-CHOP-treated patients, and a recent analysis revealed that it failed 
to recognize a subgroup with long-term survival rate of less than 50\%. ${ }^{5,6}$ Biomarkers seem to contribute to perfect riskstratification in DLBCL and to identify high-risk subgroups benefiting from intensified or novel therapies. ${ }^{7,8}$ Therefore, there arises an urgent need for biomarkers as new standard prognostic indicators not only for patients with DLBCL but also for patients with other tumors.

Several biological markers have been studied in order to recognize high-risk patients, who show an early relapse or poor response to standard treatment, but none has been proved to be completely effective, and more independent factors have still to be identified and confirmed. Proposed scoring systems are too sophisticated for daily use or their predictive value is inferior to IPI. CD40 is a $43 \mathrm{kD}$ membrane glycoprotein and a member of the tumor necrosis factor (TNF) receptor family, and it is expressed in certain cells, including B lymphocytes and antigen-presenting cells such as dendritic cells and monocytes. As we all know, CD40 is a growth signal receptor for B lymphocytes. ${ }^{9}$ Recent studies have shown that CD40 is expressed in DLBCL, and the higher its expression level, the better the prognosis of patients. ${ }^{10-12}$

In the present study, we used immunohistochemistry (IHC) to examine 186 paraffin-embedded tumor tissues from patients with DLBCLs treated with R-CHOP and correlated CD40 expression with patients' clinicopathological features and clinical outcomes.

\section{Materials and methods Patients}

Pretreatment biopsies of 186 consented patients with DLBCL admitted to the Nanjing First Hospital and Affiliated Hospital of Nantong University from 2005 to 2010 were studied, the patients met the following criteria: 1) confirmed diagnosis of DLBCL (diagnosis of de novo DLBCL in human immunodeficiency virus (HIV)-negative patients; primary mediastinal B-cell lymphoma, cutaneous LBCL, intravascular LBCL, and involvement of central nervous system at the time of diagnosis were excluded) following a pathologic review; 2) treatment with R-CHOP (rituximab, cyclophosphamide, adriamycin, vincristine, and prednisone); 3) diagnostic paraffin material detected by tissue microarray (TMA); and 4) tested negative for HIV 1. All the cases were histologically reviewed by two senior pathologists according to the World Health Organization classification of tumors of hematopoietic and lymphoid tissues to confirm the diagnosis. For germinal center B-cell-like (GCB) type, CD10+, BCL-2-, centerine+, and MUM1 are immunologically stained. For non-GCB type, CD10-, BCL-2+, centerine-, and MUM1+ are immunologically stained. Complete response
(CR), partial response (PR), stable disease, and progressive disease were defined according to International Working Group recommendations. ${ }^{13}$ Clinical follow-up data were obtained from the patients' medical records. The study was approved by the Institutional Review Boards of Nantong University (People's Republic of China) and Nanjing Medical University (People's Republic of China). The study was conducted in accordance with the Declaration of Helsinki. Written informed consent was obtained from each participant or from the patient representatives.

\section{Tissue microarray}

Tumor samples were arrayed as described previously. ${ }^{14,15}$ Slides of $5 \mu \mathrm{m}$ tissue sections were stained with hematoxylineosin stain and used to identify and mark out a representative viable tumor tissue. Using a $0.6 \mathrm{~mm}$ needle, core biopsies from the areas of corresponding paraffin-embedded blocks were placed at defined coordinates in the recipient paraffin array blocks using a manual arrayer (Beecher Instruments, Sun Prairie, WI, USA). The array blocks were constructed at a density of 80-150 cores per array. A single core was evaluated from each case. The analysis of single TMA core typically shows more than $90 \%$ concordance with the conventional whole section analysis of tumor markers and it has been validated previously. ${ }^{16}$

\section{Immunohistochemistry}

Consecutive tissue sections ( $4 \mu \mathrm{m}$ thickness) from the TMAs were cut into adhesive-coated slides and deparaffinized with serial xylene, ethanol gradient, and distilled water. Heat-induced antigen retrieval was performed in a pressure cooker containing $1 \mathrm{mM}$ ethylene diamine tetraacetic acid ( $\mathrm{pH} 8.0$ ) or $10 \mathrm{mM}$ citrate $\left(\mathrm{pH} 6.0\right.$ ) at $120^{\circ} \mathrm{C}$ for 3 minutes. Endogenous peroxidase was blocked using 10\% hydrogen peroxide solution at room temperature for 5 minutes. The sections were then rinsed in phosphate buffered saline (PBS) for 5 minutes and incubated at $4{ }^{\circ} \mathrm{C}$ overnight with monoclonal antibodies against CD40 (Santa Cruz Biotechnology, Inc., Dallas, TX, USA). After washing with PBS, two to three drops of secondary antibody were added, and the sections were incubated at room temperature for 30 minutes. Finally, the sections were counterstained with Mayer's hematoxylin and then mounted. Additional sections, running in parallel but without addition of the primary antibody in the first incubation, served as negative controls.

IHC staining was evaluated independently by two experienced pathologists, who were blinded to the clinical data. One hundred cells were evaluated in each tumor core, and the percentage of positive cells was calculated. The scores 
of the three tumor cores were finally averaged for each patient. A case was considered positive if $10 \%$ or more of the cells were stained with an antibody. Digital microscopy images were captured using an Olympus BX51 microscope (Olympus, Tokyo, Japan), imported into Image Pro Plus Version 4.5 (Media Cybernetics Inc., Bethesda, MD, USA), and processed digitally for adjustments of contrast, brightness, and color balance. The integrated optical density (IOD) of all the positive staining in each field and area of interest (AOI) were measured. The IOD was used to evaluate the area and intensity of the positive staining. The mean density (IOD/AOI) represented the concentration of specific protein per unit area. Using the median staining mean density of CD40 by IHC as the staining threshold, we separated patients into low-expression group with values $<89.27$ and highexpression group with values $\geq 89.27$.

\section{Statistical analysis}

Statistical analyses were performed with SPSS software version 11.0. Mann-Whitney $U$-test (two-tailed) was performed to compare two groups, and one-way analysis of variance (ANOVA) and Pearson correlation were performed to determine the significance of differences in multiple comparisons. Overall survival (OS) and progression-free survival (PFS) time distributions were calculated by the Kaplan-Meier method and analyzed by log-rank test. PFS was defined as the interval between the date of initial diagnosis and the date of disease progression or death from lymphoma or treatment toxicity. OS was calculated as the time from diagnosis to the date of death or last contact. Differences were considered statistically significant if $P$-values were less than 0.05 .

\section{Results}

\section{Clinical characteristics of the series}

A summary of the clinical characteristics of the entire series of patients is given in Table 1. Complete clinical and histopathologic data were available for all 186 patients. All patients received six to eight (median 6.8) cycles of standard R-CHOP regimen. The overall response rate $(\mathrm{CR}+\mathrm{PR})$ was $86.7 \%(\mathrm{CR}$ was $67.7 \%$ ), while disease was stable in $7.9 \%$ and progressed in $5.4 \%$ patients. The median follow-up time for all patients was 50.4 months. The median follow-up time for patients alive at the follow-up was 46 (6-92) months. The estimated 3 -year OS was $70.5 \%$ and the estimated PFS was $61.3 \%$.

\section{Correlation between CD40 expression and clinicopathological parameters}

About 131 patients (70.4\%) showed IHC-positive CD40. Based on Muris algorithm, 68 patients (36.5\%) were
Table I Summary of clinical characteristics of I86 patients with diffuse large B-cell lymphoma

\begin{tabular}{ll}
\hline Characteristics & Number of patients (\%) \\
\hline Age (range), years & $(16-85)$ \\
Median & 64.2 \\
$\leq 64$ & $79(42.5)$ \\
$>64$ & $107(57.5)$ \\
Sex & \\
Male & $113(60.7)$ \\
Female & $73(39.3)$ \\
Performance status & \\
0 & $33(17.7)$ \\
I & $59(31.7)$ \\
2 & $65(35)$ \\
3 & $20(10.8)$ \\
4 & $9(4.8)$ \\
Lactate dehydrogenase & $103(60.7)$ \\
Normal & $83(39.3)$ \\
High & \\
Extranodal involvement & $105(56.5)$ \\
Yes & $81(43.5)$ \\
No &
\end{tabular}

classified as GCB subtype and 63 patients (33.9\%) as nonGCB subtype. About 55 patients (29.6\%) showed IHCnegative CD40. Twenty nine patients out of 55 (15.6\%) were classified as GCB subtype and 26 patients (14\%) as non-GCB subtype. There were no statistically significant differences in DLBCL subclassification between moderate positivity (Figure 1A) and high positivity groups (Figure 1B) $(P>0.05)$. The CD40 expression was observed to correlate with age, sex, stage, lactate dehydrogenase level, and IPI score, but no statistical significances were obtained (Table 2) $(P>0.05)$.

\section{Effect of CD40 expression on therapeutic response}

Given the expression level of CD40 has an important prediction value for therapeutic response (R-CHOP), we found among 186 documented cases, CD40 was expressed in high expression group in 85 complete remissions $(67.5 \%)$ and in low expression group only in 41 cases $(32.5 \%)(P<0.05)$. 

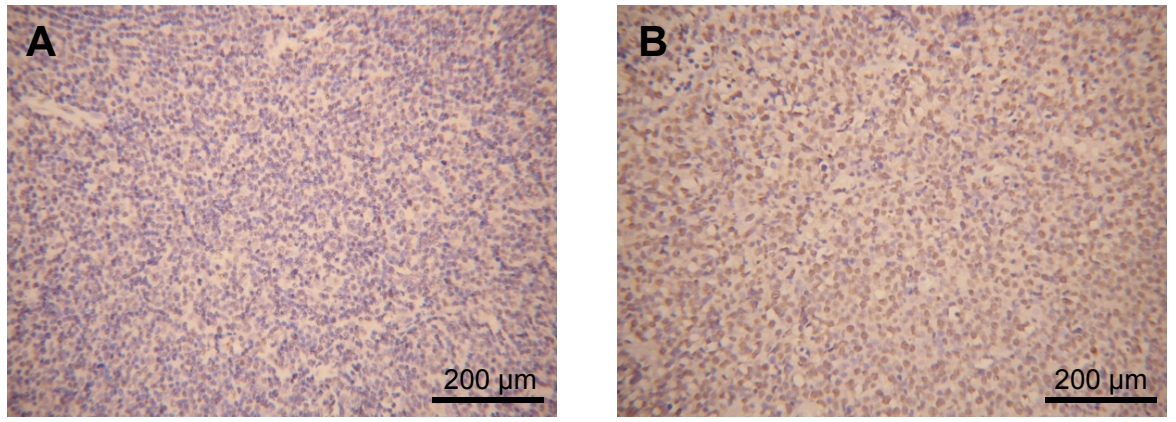

Figure I CD40 immunohistochemistry in tissues of DLBCL patients.

Notes: (A) Low and (B) high CD40 expression in a case of DLBCL $\times 200$ magnification.

Abbreviation: DLBCL, diffuse large B-cell lymphoma.

In patients with complete remission $(n=126)$, CD40 was expressed in $49.4 \%$ of the tumor cells on average $(95 \%$ confidence interval [CI], 35-89), whereas in patients without complete remission, CD40 was expressed in $18.7 \%$ of tumor cells $(95 \% \mathrm{CI}, 5-42 ; P<0.05)$. CD40 expression was the highest in patients with $\mathrm{CR}$ and higher in patients with PR compared to patients with no response $(P<0.05)$ (Figure 2). All factors associated with failure to achieve complete remission in the univariate models were involved in multivariate analysis, which showed that CD40 expression in $<35 \%$ of tumor cells $(P=0.018)$, B symptoms $(P=0.022)$, and high IPI $(P=0.009)$ were the independent predictive factors for treatment resistance.

\section{Correlation of CD40 expression with PFS and OS}

The relationships between the percentage of CD40 expression and survival rates for 186 DLBCL patients (an average of 4.1 years of follow-up) were also examined. Figure 3 shows the Kaplan-Meier survival curves of the patients grouped by the percentage of CD40-expressing cells in their tumors. The cumulative survival rate of the patients with high expression of CD40 was significantly higher compared with patients with low expression of CD40 $(P<0.05)$. Patients with high expression of CD40 lived longer compared with patients with low expression of CD40.

Table 2 Association between CD40 expression (IOD/AOI) and different clinicopathological features of diffuse large B-cell lymphoma

\begin{tabular}{|c|c|c|c|}
\hline Clinicopathological features & Number of cases & CD40 expression & $P$-value \\
\hline \multicolumn{4}{|l|}{ Age } \\
\hline$\leq 64$ & 79 & $99.42 \pm 6.94$ & \\
\hline$>64$ & 107 & $93.8 I \pm 9.35$ & 0.5362 \\
\hline \multicolumn{4}{|l|}{ Sex } \\
\hline Male & 113 & $95.33 \pm 7.87$ & \\
\hline Female & 73 & $89.57 \pm 6.24$ & 0.6129 \\
\hline \multicolumn{4}{|l|}{ Lactate dehydrogenase } \\
\hline Normal & 103 & $96.11 \pm 6.49$ & \\
\hline High & 83 & $88.96 \pm 8.42$ & 0.3985 \\
\hline \multicolumn{4}{|l|}{ Stage } \\
\hline I & 23 & $101.03 \pm 7.13$ & \\
\hline II & 49 & $92.88 \pm 6.99$ & \\
\hline III & 75 & $95.21 \pm 5.78$ & \\
\hline IV & 39 & $89.79 \pm 9.27$ & 0.2408 \\
\hline \multicolumn{4}{|l|}{ IPI } \\
\hline Low risk & 25 & $99.58 \pm 8.81$ & \\
\hline Low to intermediate risk & 31 & $94.11 \pm 5.99$ & \\
\hline Intermediate to high risk & 54 & $91.23 \pm 7.67$ & \\
\hline High risk & 76 & $87.98 \pm 10.35$ & 0.3647 \\
\hline
\end{tabular}

Notes: Data are presented as Mean \pm SD. Results are in response to a one way analysis of variance (ANOVA). $P$-values were considered significant at $<0.05$. Abbreviations: IOD, integrated optical density; AOI, area of interest; SD, standard deviation, IPI, International Prognostic Index. 


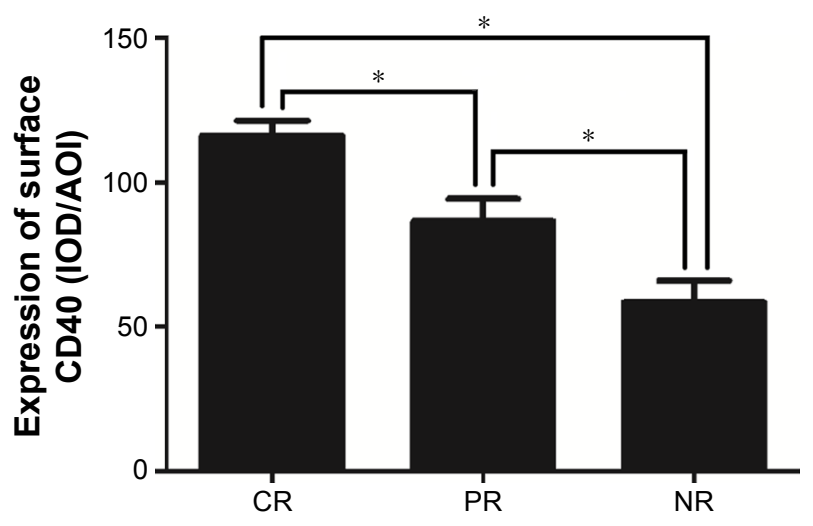

Figure 2 Mean expression of CD40 using immunohistochemistry in tumors CR, PR, NR, and control.

Notes: ${ }^{*} P<0.05$. The black lines connecting different bars represent the comparison made between the two. Comparisons were made between $C R$ with $P R$ and $C R$ vs $N R, P R$ vs NR. $P$-values were considered significant at $<0.05$.

Abbreviations: $C R$, complete response; PR, partial response; NR, no response; $I O D$, integrated optical density; $\mathrm{AOI}$, area of interest.

\section{Discussion}

The chimeric monoclonal antibody - rituximab - chooses CD20 antigen as its target in B cells. Therefore, in the treatment of DLBCL, it is an extremely important drug. Young and elderly patients benefited from the combination of $\mathrm{CHOP}$ and rituximab, and their clinical outcome significantly improved. ${ }^{17,18}$ However, there are still a considerable number of patients who show no response to rituximab-based therapy or who relapse early, and the outcome of these patients to second-line or salvage therapies is not satisfactory. The IPI remains the most successful clinical prognostic instrument for DLBCL. However, the IPI score is no longer considered as a strong prognostic indicator for R-CHOP-treated patients, and a recent analysis revealed that it failed to recognize subgroups with a long-term survival rate lower than 50\%. ${ }^{5,6}$ Biomarkers seem to contribute to perfect risk stratification in DLBCL and to identify high-risk subgroups of patients benefiting from intensified or novel therapies. ${ }^{2}$ Currently there is no a reliable system that could effectively identify such patients prospectively, which needs to identify a more effective prognostic evaluation system for patients treated with R-CHOP.

CD40 is a member of the tumor necrosis factor receptor family and is expressed on non-immune cells and on tumor cells-mostly in B-cell malignancies - as well as on several immune cells including normal B cells. ${ }^{19}$ It has been demonstrated that agonistic CD40 antibodies may mediate antitumor activity through the activation of CD40positive antigen-presenting cells, including dendritic cells. ${ }^{20,21}$ A recent report showed that in the help of CD40-independent natural killer (NK) cells, the immunity against endogenous B-cell lymphoma by dendritic cell vaccine-induced T-cell was enhanced. Hömberg et $\mathrm{al}^{22}$ showed that exogenous dendritic cells can trigger endogenous NK cells, resulting in the generation of interferon- $\gamma$ and initiating a cascade that eventually leads to cytotoxic T-lymphocyte responses. This pathway depends on a preceding maturation step to obviate CD4(+) T-cell help and CD40 signaling. ${ }^{22} \mathrm{~A}$ Phase I study of the agonistic IgG1 chimeric anti-CD40 antibody ChiLob7/4 in patients with CD40-expressing solid tumors and DLBCL was conducted. The authors found that ChiLob7/4 can activate B and NK cells at doses that can be administered safely, and ChiLob7/4 should be tested in combination with other antibodies and chemotherapy agents. ${ }^{23}$ In addition, another Phase I and Phase II study of dacetuzumab was conducted in patients with refractory or recurrent B-cell Non-Hodgkin's lymphoma (NHL), including DLBCL. ${ }^{24,25}$ CD40L/CD154 mainly expressed on activated helper $\mathrm{T}$ cells is the ligand of CD40 and interacts with it. This interaction leads to the B cells guided by their differentiation program - a process
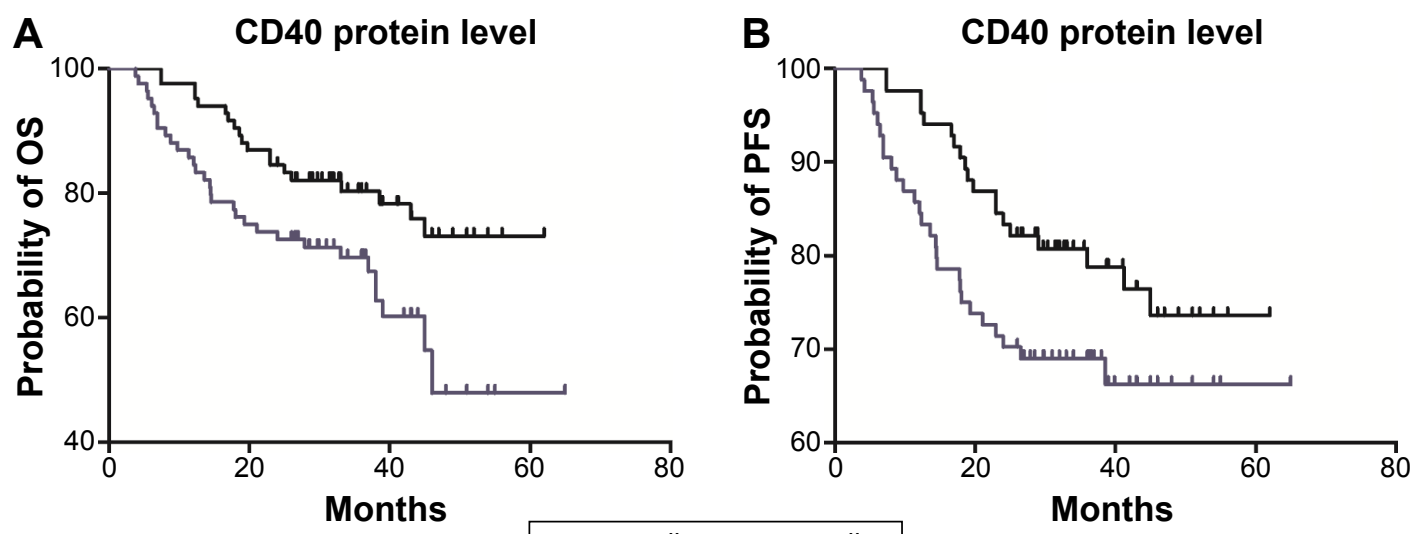

$\perp \geq$ median $\_<$median

Figure 3 Overall survival (A) and progression-free survival (B) curves of patients according to CD40 protein expression level. Note: $P<0.05$. 
that mainly takes place within the germinal centers. ${ }^{26,27}$ CD40 signal has been shown to be associated with B-cell proliferation, growth, and differentiation; however, several recent studies have shown that it plays an important role in a diverse array of biological processes. CD40 engagement may be associated with weakening the ability of survival and malignancy in tumor cells. It can promote apoptosis and stimulate angiogenesis and transcription/secretion of matrix metalloproteinase. ${ }^{28,29}$ In addition, it has been reported that CD40 upregulates the expression of ICAM- $1,{ }^{30}$ a celladhesion molecule important for cell-cell interactions. These programs are closely related to the migration and invasion of tumor cells. However, how the expressions of CD40 affect the malignant cells of DLBCL are not well established. Some studies have found that the expression of CD40 defined by IHC in DLBCL results in a favorable prognosis. ${ }^{10,11,29}$

Therefore, we analyzed the expression of CD40 in specimens of DLBCL. In our study, all the 186 patients with DLBCL were treated with R-CHOP and the expression of CD40 was found to be higher in patients with CR compared to patients with PR, and in patients with PR compared to patients with no response. Therefore, high CD40 expression can predict DLBCL patients' response to R-CHOP regime. The preliminary results demonstrate that the long-term survival rate including 3-year OS and 3-year PFS in patients with high CD40 expression was superior compared with patients with low expression. The expression level of CD40 positively correlates with the prognosis of DLBCL patients. So if we formulate more adequate regimen based on CD40 expression, the long-term survival rate can be improved in DLBCL patients with different risk factors. In order to minimize the inherent biases of the study, we selected only patients with de novo DLBCL treated with standard first-line chemotherapy and excluded other presentations of DLBCL such as HIV-positive, primary mediastinal, central nervous system, intravascular and testicular lymphomas, transformed NHL, and cutaneous LBCL. ${ }^{31}$

\section{Conclusion}

In conclusion, this study demonstrates that in routine pathologic specimens, CD40 expression can be detected by IHC. Low levels of CD40 expression indicate a poor prognosis, since R-CHOP produces limited benefits. These results have shown that we can effectively identify DLBCL patients with poor clinical outcome, which guides us to adopt more intensive treatment for those patients. Our finding was based on the analysis of a small cohort of DLBCL patients and is a retrospective study. Patients with DLBCL should be classified based on the effective prognostic factors such as CD40 and treatment including upfront hematopoietic stem cell transplantation should be aggressively administered to patients with an unfavorable prediction. To further advance the detection of CD40 expression for clinical application, larger-scale, prospective studies on DLBCL patients are required.

\section{Disclosure}

The authors declare no conflicts of interest in this work.

\section{References}

1. Lossos IS, Morgensztern D. Prognostic biomarkers in diffuse large B-Cell lymphoma. J Clin Oncol. 2006;24:995-1007.

2. Shustik J, Han G, Farinha P, et al. Correlations between BCL6 rearrangement and outcome in patients with diffuse large B-cell lymphoma treated with CHOP or R-CHOP. Haematologica. 2010;95: 96-101.

3. Sehn LH, Berry B, Chhanabhai M, et al. The revised International Prognostic Index (R-IPI) is a better predictor of outcome than the standard IPI for patients with diffuse large B-cell lymphoma treated with R-CHOP. Blood. 2007;109:1857-1861

4. A predictive model for aggressive non-Hodgkin's lymphoma. The International Non-Hodgkin's Lymphoma Prognostic Factors Project. N Engl J Med. 1993;329:987-994.

5. Goto N, Tsurumi H, Kasahara S, et al. Serum interleukin-18 level is associated with the outcome of patients with diffuse large B-cell lymphoma treated with CHOP or R-CHOP regimens. Eur J Haematol. 2011;87: 217-227.

6. Song G, Gu L, Li J, et al. Serum microRNA expression profiling predict response to R-CHOP treatment in diffuse large B-cell lymphoma patients. Ann Hematol. 2014;93:1735-1743.

7. Ott G, Ziepert M, Klapper W, et al. Immunoblastic morphology but not the immunohistochemical GCB/nonGCB classifier predicts outcome in diffuse large B-cell lymphoma in the RICOVER-60 trial of the DSHNHL. Blood. 2010;116:4916-4925.

8. Ennishi D, Asai H, Maeda Y, et al. Statin-independent prognosis of patients with diffuse large B-cell lymphoma receiving rituximab plus CHOP therapy. Ann Oncol. 2010;21:1217-1221.

9. Miyashita T, McIlraith MJ, Grammer AC, Miura Y, Attrep JF, Shimaoka Y, Lipsky PE. Bidirectional regulation of human B cell responses by CD40-CD40 ligand interactions. J Immunol. 1997;158: $4620-4633$.

10. Linderoth J, Ehinger M, Jerkeman M, et al. CD40 expression identifies a prognostically favourable subgroup of diffuse large B-cell lymphoma. Leuk Lymphoma. 2007;48:1774-1779.

11. Rydström K, Linderoth J, Nyman H, et al. CD40 is a potential marker of favorable prognosis in patients with diffuse large B-cell lymphoma treated with immunochemotherapy. Leuk Lymphoma. 2010; 51:1643-1648.

12. Rydström K, Joost P, Ehinger M, et al. Gene expression profiling indicates that immunohistochemical expression of CD40 is a marker of an inflammatory reaction in the tumor stroma of diffuse large B-cell lymphoma. Leuk Lymphoma. 2012;53:1764-1768.

13. Cheson BD, Pfistner B, Juweid ME, et al. Revised response criteria for malignant lymphoma. J Clin Oncol. 2007;25:579-586.

14. Nocito A, Kononen J, Kallioniemi OP, Sauter G. Tissue microarrays (TMAs) for high-throughput molecular pathology research. Int $J$ Cancer. 2001;94:1-5.

15. Torhorst J, Bucher C, Kononen J, et al. Tissue microarrays for the rapid linking of molecular changes to clinical endpoints. Am J Pathol. 2001;159:2249-2256. 
16. Camp RL, Charette LA, Rimm DL. Validation of tissue microarray technology in breast carcinoma. Lab Invest. 2000;80:1943-1949.

17. Pfreundschuh M, Trümper L, Osterborg A, et al. CHOP-like chemotherapy plus rituximab versus CHOP-like chemotherapy alone in young patients with good-prognosis diffuse large-B-cell lymphoma: a randomised controlled trial by the MabThera International Trial (MInT) Group. Lancet Oncol. 2006;7:379-391.

18. Sehn LH, Donaldson J, Chhanabhai M, et al. Introduction of combined CHOP plus rituximab therapy dramatically improved outcome of diffuse large B-cell lymphoma in British Columbia. J Clin Oncol. 2005; 23:5027-5033.

19. van Kooten C, Banchereau J. CD40-CD40 ligand. J Leukoc Biol. 2000;67:2-17.

20. Hassan SB, Sorensen JF, Olsen BN, Pedersen AE. Anti-CD40-mediated cancer immunotherapy: an update of recent and ongoing clinical trials. Immunopharmacol Immunotoxicol. 2014;36:96-104.

21. Vonderheide RH, Glennie MJ. Agonistic CD40 antibodies and cancertherapy. Clin Cancer Res. 2013;19:1035-1043.

22. Hömberg N, Adam C, Riedel T, et al. CD40-independent natural killer-cell help promotes dendritic cell vaccine-induced T-cell immunity against endogenous B-cell lymphoma. Int J Cancer. 2014; 135:2825-2833.

23. Johnson P, Challis R, Chowdhury F, et al. Clinical and biological effects of an agonist anti-CD40 antibody: a Cancer Research UK phase I study. Clin Cancer Res. 2015;21:1321-1328.

24. Advani R, Forero-Torres A, Furman RR, et al. Phase I study of the humanized anti-CD40 monoclonal antibody dacetuzumab in refractory or recurrent Non-Hodgkin's lymphoma. J Clin Oncol. 2009;27: 4371-4377.
25. de Vos S, Forero-Torres A, Ansell SM, et al. A phase II study of dacetuzumab (SGN-40) in patients with relapsed diffuse large B-cell lymphoma (DLBCL) and correlative analyses of patient-specific factors. J Hematol Oncol. 2014;7:44-52.

26. Ghia P, Boussiotis VA, Schultze JL, et al. Unbalanced expression of bcl-2 family proteins in follicular lymphoma: contribution of CD40 signaling in promoting survival. Blood. 1998;91:244-251.

27. Voorzanger-Rousselot N, Favrot M, Blay JY. Resistance to cytotoxic chemotherapy induced by CD40 ligand in lymphoma cells. Blood. 1998;92:3381-3387.

28. Shi XY, Dornan D. To respond or not to respond to CD40 agonism. Oncoimmunology. 2012;1:83-85.

29. Linderoth J, Jerkeman M, Cavallin-Ståhl E, Kvaløy S, Torlakovic E; Nordic Lymphoma Group Study. Immunohistochemical expression of CD23 and CD40 may identify prognostically favorable subgroups of diffuse large B-cell lymphoma: a Nordic Lymphoma Group Study. Clin Cancer Res. 2003;9:722-728.

30. Lee HH, Dempsey PW, Parks TP, Zhu X, Baltimore D, Cheng G. Specificities of CD40 signaling: involvement of TRAF2 in CD40-induced NF-B activation and intercellular adhesion molecule-1 up-regulation. Proc Natl Acad Sci U S A. 1999;96:1421-1426.

31. Hao X, Wei X, Huang F, et al. The expression of CD30 based on immunohistochemistry predicts inferior outcome in patients with diffuse large B-cell lymphoma. PLoS One. 2015;10:e0126615.
OncoTargets and Therapy

\section{Publish your work in this journal}

OncoTargets and Therapy is an international, peer-reviewed, open access journal focusing on the pathological basis of all cancers, potential targets for therapy and treatment protocols employed to improve the management of cancer patients. The journal also focuses on the impact of management programs and new therapeutic agents and protocols on

\section{Dovepress}

patient perspectives such as quality of life, adherence and satisfaction. The manuscript management system is completely online and includes a very quick and fair peer-review system, which is all easy to use. Visit http://www.dovepress.com/testimonials.php to read real quotes from published authors. 